\title{
Thomas point process in pulse, particle, and photon detection
}

\author{
Kuniaki Matsuo, Malvin Carl Teich, and Bahaa E. A. Saleh
}

\begin{abstract}
Multiplication effects in point processes are important in a number of areas of electrical engineering and physics. We examine the properties and applications of a point process that arises when each event of a primary Poisson process generates a random number of subsidiary events with a given time course. The multiplication factor is assumed to obey the Poisson probability law, and the dynamics of the time delay are associated with a linear filter of arbitrary impulse response function; special attention is devoted to the rectangular and exponential case. Primary events are included in the final point process, which is expected to have applications in pulse, particle, and photon detection. We refer to this as the Thomas point process since the counting distribution reduces to the Thomas distribution in the limit of long counting times. Explicit results are obtained for the singlefold and multifold counting statistics (distribution of the number of events registered in a fixed counting time), the time statistics (forward recurrence time and interevent probability densities), and the counting correlation function (noise properties). These statistics can provide substantial insight into the underlying physical mechanisms generating the process. An example of the applicability of the model is provided by betaluminescence photons produced in a glass photomultiplier tube, when Cherenkov events are also present.
\end{abstract}

\section{Introduction}

To describe the statistics of photons, particles, or in general a random sequence of pulses, the theory of point processes is used. The simplest and most common process is the homogeneous Poisson point process (HPP). It is characterized by a single quantity, its rate, which is constant. One of its distinguishing features is that it evolves in time without aftereffects. This means that the occurrence times, and number of events before an arbitrary time, have no bearing on the subsequent occurrence times and number of events. It is said to have zero memory. ${ }^{1}$ Photons of a stabilized laser above threshold obey the HPP to very good approximation.

There are many generalizations of the HPP. One case that has been studied extensively is the doubly stochastic Poisson point process (DSPP), which differs from the HPP in that the rate is no longer constant. ${ }^{2}$ Rather it takes on a stochastic nature of its own. This process was first examined by Cox (and by Bartlett in the discussion to Cox's paper). ${ }^{3}$ The designation DSPP was introduced to emphasize that two kinds of randomness occur: randomness associated with the

The authors are with Columbia University, Department of Electrical Engineering, Columbia Radiation Laboratory, New York, New York 10027.

Received 14 January 1983.

0003-6935/83/121898-12\$01.00/0.

(C) 1983 Optical Society of America.
Poisson point process itself and an independent randomness associated with its rate. Photons of classical sources of light are described by the DSPP.

A special case of the DSPP obtains when the stochastic rate is a filtered HPP (i:e., shot noise); it is, therefore, convenient to call this the shot-noise-driven doubly stochastic Poisson point process (SNDP). ${ }^{4-8}$ The SNDP arises when the rate of a (secondary) inhomogeneous Poisson pulse sequence is determined by another (primary) Poisson pulse sequence. An example is the sequence of primary Poisson electrons producing a sequence of photons in betaluminescence. The SNDP has been recently studied in great detail. The singlefold and multifold counting and time statistics, ${ }^{8}$ as well as the time statistics in the presence of dead time and sick time, ${ }^{7}$ have been obtained. One important result that emerges from the study is that in the limit of counting time long in comparison with the fluctuation time of the shot noise, a unique SNDP counting distribution emerges, the Neyman type- $A$ distribution. ${ }^{9-11}$ Even in the nonstationary case, this limit turns out to be appropriate. ${ }^{12}$ The behavior of the SNDP in the presence of dead time (self-excitation) has also been examined in several cases $7,13,14$; phenomenological arguments show that in certain limits, the Neyman type- $A$ counting distribution provides a satisfactory approximation in this case as well. ${ }^{14}$ Finally, multiplied-Poisson point processes have been examined in the context of quantum optics, where interference effects can occur, ${ }^{15}$ and the counting statistics for cascades of SNDPs have been formulated. ${ }^{16}$ 
About thirty-five years ago, Thomas carried out a study ${ }^{17}$ in the context of ecology in which she introduced a two-parameter counting distribution distinct from the Neyman type- $A$ only in that primary events were included in the final counts. Although she originally referred to this as the double-Poisson distribution, it has since come to be called the Thomas distribution. In this paper, we consider a stochastic point process that includes time dynamics and reduces to the Thomas counting distribution in the limit of long counting times. We assume that the primary events are instantaneously carried forward to the final process and that the secondary events are randomly delayed with respect to the primary events. The model is, therefore, identical to the SNDP considered earlier with the additional condition that primary events are fed forward. This is not a trivial addition, however, since the primary and secondary events are not independent.

In Sec. II, we review the properties of the instantaneous Thomas process (when time dynamics are absent). The effects of time dynamics are incorporated in Sec. III; the counting statistics, counting correlation function, and time statistics are obtained, and their behaviors are investigated in special cases. Applications of the Thomas process are discussed in Sec. IV, and the conclusion is provided in Sec. V.

\section{Instantaneous Thomas Process}

In this section, we briefly review the Thomas distribution and study its various statistical properties. Consider the primary point process whose events are illustrated in Fig. 1(a). The number of events (counts) within a time interval $[0, T]$ is a discrete random variable $M$ having a moment generating function (mgf) $Q_{M}(s)$ $=\langle\exp (-s M)\rangle$. The angular brackets represent an ensemble average. Let each primary event produce independently $A$ subsidiary events [as illustrated in Fig. 1(b)], where $A$ is a discrete random variable that has an $\operatorname{mgf} Q_{A}(s)=\langle\exp (-s A)\rangle$. Since the primary process is fed forward and added to the secondary events, the total number of events $N$ is given by

$$
N=\sum_{k=1}^{M} A_{k}+M,
$$

where the $A_{k}$ are independent values of $A$. If $A$ and $M$ are statistically independent, it can be shown that the mgf of $N, Q_{N}(s)$, is given by ${ }^{1,11}$

$$
Q_{N}(s)=Q_{M}\left[s-\ln Q_{A}(s)\right] .
$$

This equation can be used to relate the moments of $N$ to those of $M$ and $A$. The means are related by

$$
\langle N\rangle=\langle M\rangle(1+\langle A\rangle),
$$

the second factorial moment obeys the equation

$$
\langle N(N-1)\rangle=(1+\langle A\rangle)^{2}\langle M(M-1)\rangle+\langle M\rangle\langle A(A-1)\rangle,
$$

and the variances are expressed as

$$
\operatorname{var}(N)=(1+\langle A\rangle)^{2} \operatorname{var}(M)+\langle M\rangle \operatorname{var}(A) .
$$

Equation (5) is related to the Burgess variance theorem. ${ }^{18-20}$

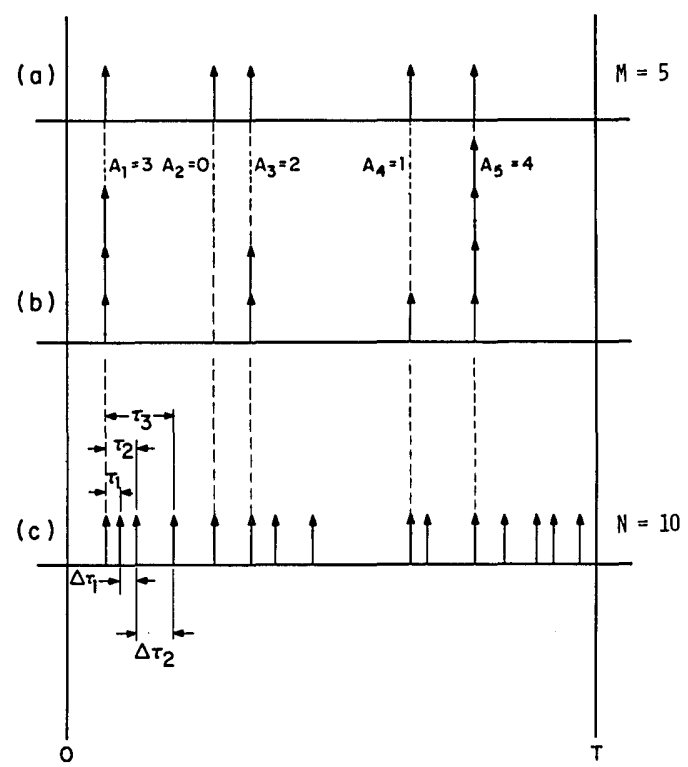

Fig. 1. Random multiplication of events: (a) primary events; (b) subsidiary events; (c) primary events plus randomly delayed subsidiary events.

When the primary events constitute a Poisson point process, the random variable $M$ has a Poisson distribution with the mgf

$$
Q_{M}(s)=\exp \langle\langle M\rangle[\exp (-s)-1]\},
$$

which, when combined with Eq. (2), gives rise to

$$
Q_{N}(s)=\exp \left\{\langle M\rangle\left[\exp (-s) Q_{A}(s)-1\right]\right\}
$$

The count variance associated with Eq. (7) is then

$$
\operatorname{var}(N)=\left(1+c_{1}\right)\langle N\rangle,
$$

where

$$
c_{1}=\langle A\rangle+\frac{\operatorname{var}(A)}{1+\langle A\rangle} .
$$

Equation (8) demonstrates that for a Poisson primary process, whatever the distribution of the multiplication factor $A$, the variance of $N$ is always proportional to the mean $\langle N\rangle$. The ratio $\operatorname{var}(N) /\langle N\rangle$ is known as the dispersion ratio or the Fano factor. 8,21 We denote the parameter $c_{1}$ as the excess Fano factor.

When the multiplication factor $A$ also has a Poisson distribution, Eq. (7) yields

$$
Q_{N}(s)=\exp [\langle M\rangle(\exp \{-s+\langle A\rangle[\exp (-s)-1]\}-1)],
$$

which is the mgf for the Thomas distribution.11,17 Using Eq. (10), we have derived the following recurrence relation for the $m$ th ordinary moment of $N$ denoted by $\left\langle N^{m}\right\rangle$ :

$$
\begin{aligned}
\left\langle N^{m+1}\right\rangle & =\langle M\rangle \sum_{k=0}^{m}\left(\begin{array}{l}
m \\
k
\end{array}\right)\left\langle N^{m-k}\right\rangle d_{k+1}, \quad\left\langle N^{0}\right\rangle=1, \\
d_{k+1} & =(1+\langle A\rangle) d_{k}+\langle A\rangle \sum_{r=1}^{k}\left(\begin{array}{l}
k \\
r
\end{array}\right) d_{k-r}, \\
d_{0} & =1 .
\end{aligned}
$$

The first three ordinary moments are explicitly written as 


$$
\begin{aligned}
\langle N\rangle= & \langle M\rangle(1+\langle A\rangle), \\
\left\langle N^{2}\right\rangle= & \langle M\rangle\left[(1+\langle M\rangle)(1+\langle A\rangle)^{2}+\langle A\rangle\right], \\
\left\langle N^{3}\right\rangle= & \langle M\rangle\left[\left(1+3\langle M\rangle+\langle M\rangle^{2}\right)(1+\langle A\rangle)^{2}\right. \\
& +3\langle A\rangle(1+\langle M\rangle)(1+\langle A\rangle)+\langle A\rangle] .
\end{aligned}
$$

The variance is obtained from Eq. (12):

$$
\operatorname{var}(N)=\langle M\rangle\left(1+3\langle A\rangle+\langle A\rangle^{2}\right),
$$

in accord with the results in Refs. 11 and 17.

The $m$ th factorial moments $F_{m}$ are obtained from the factorial moment generating function:

$$
Q_{N}^{f}(s)=\left\langle(1-s)^{N}\right\rangle,
$$

which is related to the ordinary mgf by

$$
Q_{N}^{f}(s)=\left.Q_{N}(s)\right|_{s=-\ln (1-s)} .
$$

Combining Eqs. (10) and (15) yields

$$
Q_{N}^{f}(s)=\exp \langle\langle M\rangle[(1-s) \exp (-\langle A\rangle s)-1]\} \text {. }
$$

By use of the formula ${ }^{22}$

$$
F_{m}=\left.(-1)^{m} \frac{\partial^{m}}{\partial s^{m}} Q_{N}^{f}(s)\right|_{s=0},
$$

together with Eq. (16), the $m$ th factorial moment is expressed as the recurrence relation:

$$
\begin{aligned}
F_{m+1} & =\langle N\rangle \sum_{k=0}^{m}\left(\begin{array}{l}
m \\
k
\end{array}\right) F_{m-k} b_{k+1}, \\
F_{0} & =1,
\end{aligned}
$$

where

$$
b_{k}=\frac{\langle A\rangle^{k-1}}{1+\langle A\rangle}\{\langle A\rangle+k\} .
$$

The first three factorial moments are easily shown to be

$$
\begin{aligned}
& F_{1}=\langle N\rangle, \\
& F_{2}=\langle N\rangle\left\{\langle N\rangle+\langle A\rangle \frac{2+\langle A\rangle}{1+\langle A\rangle}\right\}, \\
& F_{3}=\langle N\rangle\left\{\langle N\rangle^{2}+3\langle A\rangle\langle N\rangle \frac{2+\langle A\rangle}{1+\langle A\rangle}+\langle A\rangle^{2} \frac{3+\langle A\rangle}{1+\langle A\rangle}\right\} .
\end{aligned}
$$

The Fano factor $F_{T}$ for the case of a Poisson primary process with Poisson multiplication is simply

$$
F_{T}=\frac{\operatorname{var}(N)}{\langle N\rangle}=\frac{1+3\langle A\rangle+\langle A\rangle^{2}}{1+\langle A\rangle} .
$$

The counting distribution $p(n)$ can be obtained from the formula 23

$$
p(n)=\left.\frac{1}{n !} \frac{\partial^{n}}{\partial z^{n}} G_{N}(z)\right|_{z=0},
$$

where $G_{N}(z)$ is the probability-generating function defined as

$$
G_{N}(z)=-\left\langle z^{N}\right\rangle .
$$

This is related to the ordinary mgf by

$$
G_{N}(z)=\left.Q_{N}(s)\right|_{s=-\ln z} .
$$

Combining Eqs. (10) and (23) yields

$$
G_{N}(z)=\exp (\langle M\rangle\langle z \exp [\langle A\rangle(z-1)]-1\}) .
$$

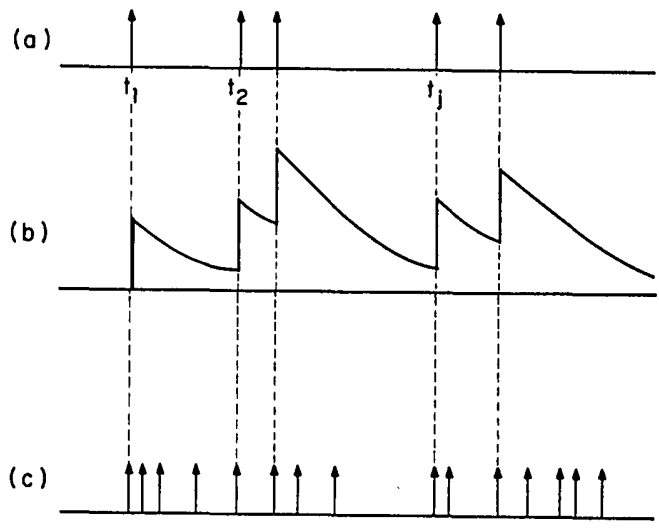

Fig. 2. Production of Thomas events: (a) primary Poisson events; (b) filtered Poisson events (shot noise); (c) primary events plus subsidiary doubly stochastic Poisson events whose rate is shot noise.

By use of Eqs. (21) and (24), the counting distribution is expressed as

$$
(n+1) p(n+1)=\langle N\rangle \sum_{k=0}^{n} c(k) p(n-k), \quad n=0,1, \ldots,
$$

$$
p(0)=\exp (-\langle M\rangle)
$$

where

$$
c(k)=\frac{1+k}{1+\langle A\rangle} \frac{\langle A\rangle^{k} \exp (-\langle A\rangle)}{k !},
$$

which can also be written as the finite sum ${ }^{11,17}$

$$
p(n+1)=\exp (-\langle M\rangle) \sum_{k=1}^{n+1} \frac{(k\langle A\rangle)^{n+1-k} \exp (-\langle A\rangle k)}{(n+1-k) !} \frac{\langle M\rangle^{k}}{k !} .
$$

In the next section, we incorporate the effects of time dynamics, demonstrating that the counting distribution reduces to the Thomas in the limit of long counting times.

\section{Thomas Process with Random Time Delay}

In the previous section, each primary event was assumed to instantaneously excite a random number of subsidiary events. In many physical systems, a time delay will be inherent in the multiplication process, and that time delay will itself be random, as illustrated in Fig. 1(c). The primary events are included in the final process for the Thomas process. The same result can be obtained by application of the SNDP, ${ }^{8}$ as shown in Fig. 2. The subsidiary events now form a DSPP whose stochastic rate is the shot noise.

The generation of the Thomas point process is represented in block diagram form in Fig. 3. A process of Poisson impulses $Z_{p}(t)$ of constant rate $\mu$ is filtered by a time-invariant linear filter with a non-negative impulse response function $h(t)$. This results in the shot-noise process $X(t) .{ }^{24}$ This process in turn is the stochastic rate giving rise to the DSPP $U(t)$. The union of the doubly stochastic Poisson point process [rate 
$X(t)]$ and the homogeneous Poisson point process (rate $\mu$ ) produces the Thomas process $Z(t)$. It is important to note that the resulting point process may not be a DSPP because $U(t)$ and $Z_{p}(t)$ are not independent.

We are interested in determining the following statistical properties of the Thomas process: (1) the counting statistics (probability distribution and moments) of the number of counts $N$ registered in the time interval $[0, T] ;(2)$ the autocorrelation function of the counts in the time interval $T$, separated by a time delay $\tau$; and (3) the time statistics, i.e., the probability density functions for the forward recurrence time and the interevent time. We begin with the moment generating functional, specializing our results as we proceed with the calculations.

\section{A. Moment Generating Functional}

We define the moment generating functional of the random process ${ }^{2,22} Z(t)$ as

$$
L_{Z}(s)=\left\langle\exp \left[-\int_{-\infty}^{\infty} Z(t) s(t) d t\right]\right\rangle \text {. }
$$

It can be shown (see Appendix) that Eq. (26) can be rewritten as

$$
\begin{aligned}
L_{Z}(s)= & \left\langle\operatorname { e x p } \left(-\int_{-\infty}^{\infty} Z_{p}(t)(s(t)\right.\right. \\
& \left.\left.\left.-\int_{-\infty}^{\infty} h(\tau-t)\{\exp [-s(\tau)]-1\} d \tau\right) d t\right)\right) \\
= & L_{Z_{p}}\left(s(t)-\int_{-\infty}^{\infty} h(\tau-t)\{\exp [-s(\tau)]-1\} d \tau\right),
\end{aligned}
$$

where the moment generating functional for the process $Z_{p}(t)$ is

$$
L_{Z_{p}}(s)=\exp \left(\mu \int_{-\infty}^{\infty}\{\exp [-s(t)]-1\} d t\right) .
$$

Combining Eqs. (27) and (28) yields the final result

$$
\begin{aligned}
L_{Z}(s)= & \exp \left\{\mu \int _ { - \infty } ^ { \infty } \left[\operatorname { e x p } \left(-s(t)+\int_{-\infty}^{\infty} h(\tau-t)\right.\right.\right. \\
& \times\{\exp [-s(\tau)]-1\} d \tau)-1] d t\}
\end{aligned}
$$

\section{B. Multifold Moment Generating Function}

The joint statistical properties of the number of counts $N_{j}$ in the $L$ time intervals $\left(t_{j}, t_{j}+T_{j}\right), j=$ $1,2, \ldots, L$, are determined from

$$
Q_{\mathbf{N}}(\mathbf{s})=\left.L_{Z}(\mathbf{s})\right|_{\mathbf{s}(t)=\mathbf{s} \mathbf{v}^{*}},
$$

where

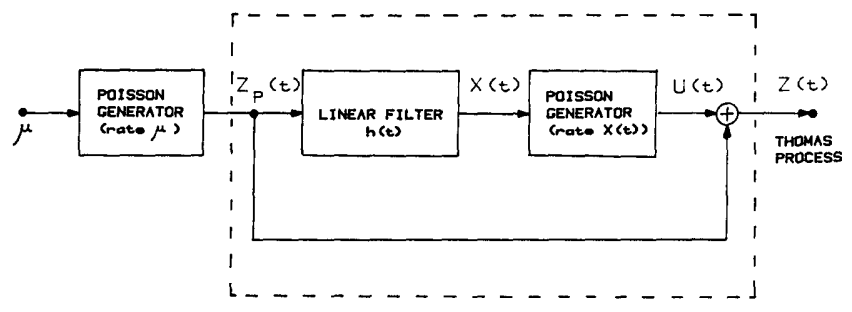

Fig. 3. Model for generation of the Thomas point process.

$$
\begin{aligned}
\mathbf{N} & =\left(N_{1}, N_{2}, \ldots, N_{L}\right), \\
\mathbf{s} & =\left(s_{1}, s_{2}, \ldots, s_{L}\right), \\
\mathbf{v}(t) & =\left[v_{1}(t), v_{2}(t), \ldots, v_{L}(t)\right], \\
v_{j}(t) & =u\left(t-t_{j}\right)-u\left(t-t_{j}-T_{j}\right) .
\end{aligned}
$$

Here $u(t)$ is the unit step function, and the asterisk * represents the operation of vector transposition. The substitution of Eq. (29) into Eq. (30) yields the $L$-dimensional moment generating function

$$
\begin{aligned}
Q_{\mathbf{N}}(\mathbf{s})= & \exp \left\{\mu \int _ { - \infty } ^ { \infty } \left[\operatorname { e x p } \left(-\sum_{j=1}^{L} s_{j} v_{j}(t)+\int_{-\infty}^{\infty} h(\tau-t)\right.\right.\right. \\
& \left.\left.\left.\times\left\{\exp \left[-\sum_{j=1}^{L} s_{j} v_{j}(\tau)\right]-1\right\} d \tau\right)-1\right] d t\right\} .
\end{aligned}
$$

By using this equation, we can explicitly determine the joint statistics of $N_{j}$.

\section{Singlefold Moment Generating Function and Moments}

The singlefold moment generating function can be found by simply substituting $L=1$ in Eq. (31). This gives

$$
\begin{aligned}
Q_{N}(s)= & \exp \left[\mu \int_{-T}^{0}\left(\exp \left\{-s+[\exp (-s)-1] h_{T}(t)\right\}-1\right) d t\right. \\
& \left.+\mu \int_{0}^{\infty}\left(\exp \left\{[\exp (-s)-1] h_{T}(t)\right\}-1\right) d t\right]
\end{aligned}
$$

where the linear filter impulse response function $h_{T}(t)$ is obtained by convolving $h(t)$ with a rectangular impulse response function on the time interval $[0, T]$. This corresponds to ideal integration (which is assumed to be noncausal for convenience), so that

$$
h_{T}(t)=\int_{0}^{T} h\left(t+t^{\prime}\right) d t^{\prime} .
$$

It can be shown that substituting $h(t)=\langle A\rangle \delta(t)$ into Eq. (32) yields Eq. (10) with $\langle M\rangle=\mu T$. This explicitly demonstrates that all moments of the Thomas instantaneous multiplication process can be recovered in the limit $T / \tau_{p} \gg 1$, where $\tau_{p}$ is the characteristic decay time for the impulse response function $h(t)$. Using Eq. (32), we obtain the following relation for the $m$ th ordinary moments of $N$ denoted $\left\langle N^{m}(T)\right\rangle$ :

$$
\left\langle N^{m+1}(T)\right\rangle=\langle N(T)\rangle \sum_{k=0}^{m}\left(\begin{array}{l}
m \\
k
\end{array}\right)\left\langle N^{m-k}(T)\right\rangle D_{k}, \quad\left\langle N^{0}(T)\right\rangle=1,
$$

where

$$
\begin{aligned}
D_{k} & =\frac{1}{1+\alpha} \frac{1}{T}\left[\int_{-T}^{0} H_{k+1}(t) d t+\int_{0}^{\infty} E_{k+1}(t) d t\right], \\
H_{k+1}(t) & =\left[1+h_{T}(t)\right] H_{k}(t)+h_{T}(t) \sum_{r=1}^{k}\left(\begin{array}{l}
k \\
r
\end{array}\right) H_{k-r}(t), \quad H_{0}=1 \\
E_{k+1}(t) & =h_{T}(t) \sum_{r=0}^{k}\left(\begin{array}{l}
k \\
r
\end{array}\right) E_{k-r}(t), \quad E_{0}=1
\end{aligned}
$$

The mean and variance are given by

$$
\langle N(T)\rangle=\mu T(1+\alpha),
$$




$$
\operatorname{var}[N(T)]=\mu T\left(1+\frac{3 \alpha}{\mathcal{M}^{\prime}}+\frac{\alpha^{2}}{M}\right)
$$

where

$$
\begin{aligned}
\mathcal{M}^{-1} & =\frac{1}{\alpha^{2} T} \int_{-\infty}^{\infty} h_{T}^{2}(t) d t, \\
\mathcal{M}^{\prime-1} & =\frac{1}{\alpha T} \int_{-T}^{0} h_{T}(t) d t+\frac{1}{3 \alpha T} \int_{0}^{\infty} h_{T}(t) d t, \\
\alpha & =\int_{0}^{\infty} h(t) d t .
\end{aligned}
$$

Here $\mathcal{M}$ and $\mathcal{M}^{\prime}$ are degrees-of-freedom parameters which depend on the counting time $T$ and the characteristic decay time $\tau_{p}$ of the impulse response function $h(t)$. The Fano factor is, therefore,

$$
F_{T}=\frac{1}{1+\alpha}\left(1+\frac{3 \alpha}{M^{\prime}}+\frac{\alpha^{2}}{M}\right) .
$$

It has previously been shown that the Fano factor for the SNDP is given by ${ }^{8}$

$$
F_{\mathrm{SNDP}}=1+(\alpha / \mathcal{M}) \text {, }
$$

where $\mathcal{M}$ is given in Eq. (35a). In the limit when $h(t)$ $=\langle A\rangle \delta(t)$, and $\langle A\rangle=\alpha, M=M^{\prime}=1$, and Eq. (20) is reproduced.

The behavior of $\mathcal{M}$ and $\mathcal{M}^{\prime}$ has been studied in detail for the cases of exponential and rectangular impulse response (filter) functions. When the filter is exponential, ${ }^{6,8}$

$$
h(t)=\frac{2 \dot{\alpha}}{\tau_{p}} \exp \left(-2 t / \tau_{p}\right) u(t),
$$

and we obtain

$$
\begin{aligned}
\mathcal{M} & =2 \beta /[\exp (-2 \beta)+2 \beta-1], \\
\mathcal{M}^{\prime} & =3 \beta /[\exp (-2 \beta)+3 \beta-1],
\end{aligned}
$$

where $\beta=T / \tau_{p}$. When $h(t)$ is rectangular, i.e.,

$$
h(t)= \begin{cases}\alpha / \tau_{p} & 0 \leq t \leq \tau_{p}, \\ 0 & \text { otherwise, }\end{cases}
$$

the degrees-of-freedom parameters turn out to be

$$
\begin{aligned}
\mathcal{M} & = \begin{cases}1 /\left(\beta-\beta^{2} / 3\right) & \beta \leq 1, \\
\beta /(\beta-1 / 3) & \beta \geq 1,\end{cases} \\
\mathcal{M}^{\prime} & = \begin{cases}3 /(1+\beta) & \beta \leq 1, \\
\beta /(\beta-1 / 3) & \beta \geq 1 .\end{cases}
\end{aligned}
$$

The degrees-of-freedom parameters $\mathcal{M}$ and $\mathcal{M}^{\prime}$ are presented graphically in Fig. 4 for the exponential $\left(\mathcal{M}_{\text {exp }}, M_{\text {exp }}^{\prime}\right)$ and rectangular $\left(\mathcal{M}_{\text {rect }}, \mathcal{M}_{\text {rect }}^{\prime}\right)$ impulse response functions.

For $\beta \ll 1, M_{\text {exp }}=M_{\text {rect }}=1 / \beta$, and $M_{\text {exp }}^{\prime}=M_{\text {rect }}^{\prime}=3$, as is evident from Eqs. (40), (41), (43), and (44). In this limit, $F_{T} \rightarrow 1$, as is seen from Eq. (37). For $\beta \gg 1$, all curves approach unity, indicating that the Fano factor achieves its maximum value

$$
F_{T}=\left(1+3 \alpha+\alpha^{2}\right) /(1+\alpha),
$$

associated with the Thomas instantaneous multiplication distribution. ${ }^{11,17}$ Note that this occurs in the limit of large counting time and is independent of the functional form of $h(t)$. Furthermore, for $\beta>1, \mathcal{M}_{\text {rect }}$ and $\mathcal{M}_{\text {rect }}^{\prime}$ are identical.
From Fig. 4, it is apparent that the specific shape of the filter function plays its largest role in the vicinity of $\beta=1$. This is to be expected, since for $\beta \ll 1$ the process approaches Poisson, while for $\beta \gg 1$ it approaches the Thomas instantaneous process. These limits are valid for arbitrary $h(t)$. Like the SNDP, the Thomas process is particlelike in nature. For short counting times, the particlelike clusters are cut apart leading to the independence characteristic of the Poisson, whereas for long counting times they are fully captured. ${ }^{6}$ This is in sharp distinction to the counting process obtained for thermal light, which is wavelike in nature. ${ }^{6}$

\section{Factorial Moments and Counting Distribution}

The factorial moments and the counting distribution can be derived from the factorial moment generating function defined by Eq. (14). This is related to the ordinary mgf in accordance with Eq. (15). The substitution of Eq. (32) into Eq. (15) yields the factorial moment generating function for the Thomas process

$$
\begin{aligned}
Q_{N}^{f}(s)= & \exp \left(\mu \int_{-T}^{0}\left\{(1-s) \exp \left[-s h_{T}(t)\right]-1\right\} d t\right. \\
& \left.+\mu \int_{0}^{\infty}\left\{\exp \left[-s h_{T}(t)\right]-1\right\} d t\right) .
\end{aligned}
$$

By use of Eq. (17), a straightforward calculation provides the following recurrence equation for the $m$ th factorial moments:

$$
F_{m+1}=\langle N\rangle \sum_{k=0}^{m}\left(\begin{array}{l}
m \\
k
\end{array}\right) F_{m-k} B_{k+1},
$$

where

$$
\begin{aligned}
F_{0}= & 1, \\
B_{k}= & \frac{1}{T(1+\alpha)}\left\{\int_{-T}^{0}\left[h_{T}(t)+k\right]\left[h_{T}(t)\right]^{k-1} d t\right. \\
& \left.+\int_{0}^{\infty}\left[h_{T}(t)\right]^{k} d t\right\} .
\end{aligned}
$$

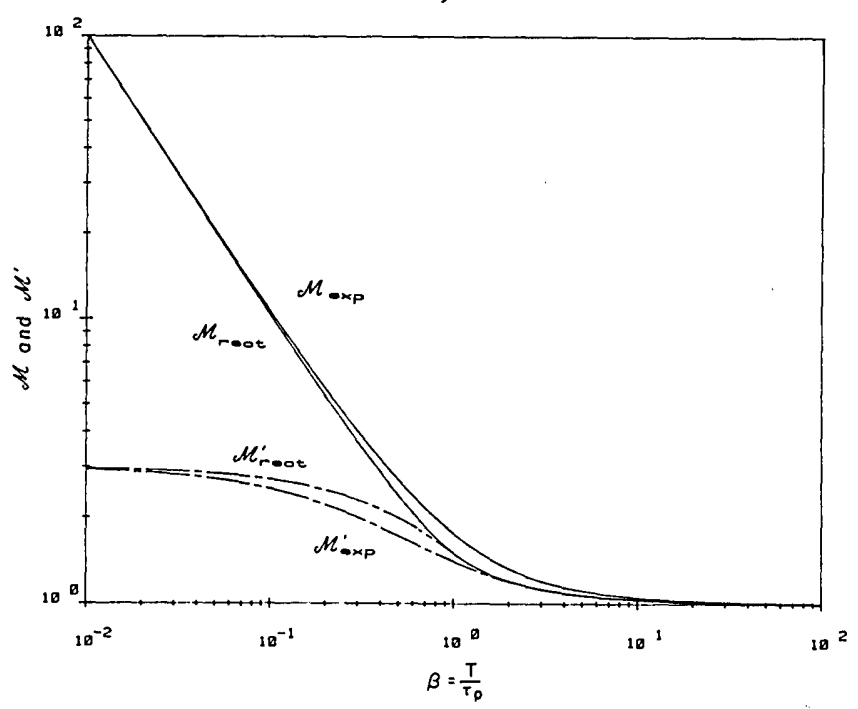

Fig. 4. Dependence of the degrees-of-freedom parameters $M$ and $\mathcal{M}^{\prime}$ on the ratio $\beta=T / \tau_{p} . \quad M_{\text {exp }}$ and $\mathcal{M}_{\text {exp }}^{\prime}$ are for the exponential case, whereas $M_{\text {rect }}$ and $M_{\text {rect }}^{\prime}$ are for the rectangular case. Observe that $\mathcal{M}_{\text {exp }}=\mathcal{M}_{\text {rect }}=\tau_{p} / T$ and $\mathcal{M}_{\text {exp }}^{\prime}=\mathcal{M}_{\text {rect }}^{\prime}=3$ for $T / \tau_{p} \ll 1$, whereas $\mathcal{M}_{\text {exp }}$ $=M_{\text {rect }}=M_{\text {exp }}^{\prime}=M_{\text {rect }}^{\prime}=1$ for $T / \tau_{p} \gg 1$. 


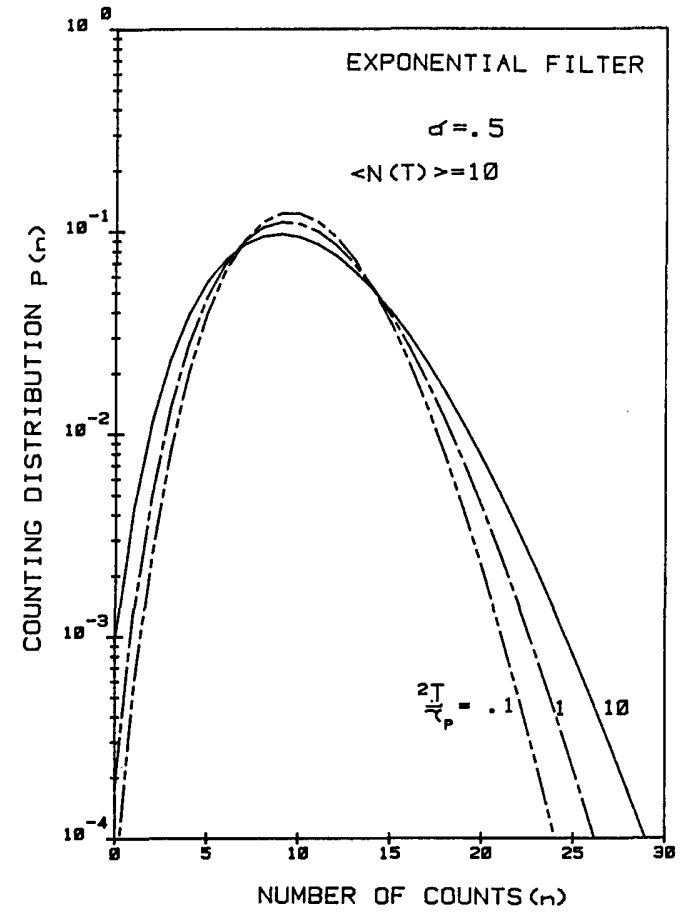

(a)

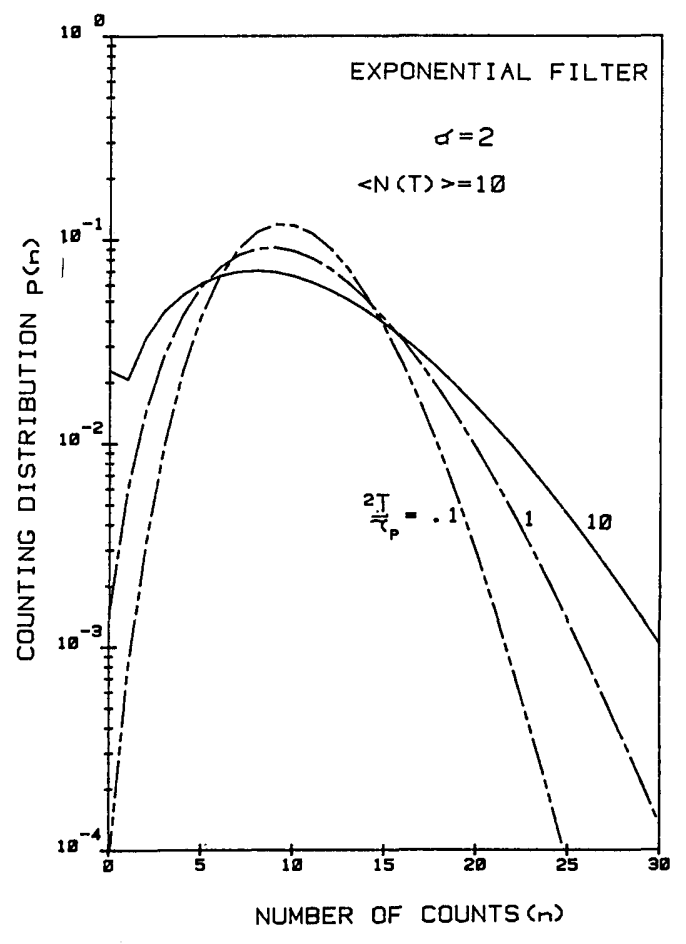

(b)

Fig. 5. Counting distribution $p(n)$ vs count number $n$, with $2 T / \tau_{p}$ as a parameter, for the Thomas process. The impulse response function for the filter is exponential with time constant $\tau_{p} / 2$. In all cases the mean number of events in the counting time $T$ is $\langle N(T)\rangle=10$. (a) multiplication parameter $\alpha=0.5$; (b) multiplication parameter $\alpha=2.0$.

It can be shown that Eq. (47) reduces to Eq. (18) provided $\langle M\rangle=\mu T, h(t)=\langle A\rangle \delta(t)$, and $\langle A\rangle=\alpha$.

The probability distribution for the number of counts in the interval $[0, T]$ can be computed by use of Eq. (21). Alternatively, it can be obtained from Eq. (46) by means of the relation

$$
p(n)=\left.(-1)^{n}\left(\partial^{n} / \partial s^{n}\right) Q_{N}^{f}(s)\right|_{s=1} .
$$

This leads to a recurrence relation for the counting distribution of the form

$$
\begin{aligned}
(n+1) p(n+1) & =\langle N\rangle \sum_{k=0}^{n} C(k) p(n-k), \\
p(0) & =\exp \left[\frac{\langle N\rangle}{1+\alpha}\left(\frac{1}{T} \int_{0}^{\infty}\left\{\exp \left[-h_{T}(t)\right]-1\right\} d t-1\right)\right],
\end{aligned}
$$

where

$$
\begin{aligned}
C(k) & =\frac{1}{k !} \frac{1}{(1+\alpha) T}\left[(k+1) \int_{-T}^{0} J_{k}(t) d t+\int_{0}^{\infty} J_{k+1}(t) d t\right], \\
J_{k}(t) & =\left[h_{T}(t)\right]^{k} \exp \left[-h_{T}(t)\right] .
\end{aligned}
$$

Equations (50a) and (50b) are identical to Eqs. (25a) and (25b), respectively, when $T / \tau_{p} \gg 1$ and $\mu T=$ $\langle M\rangle$.

In Fig. 5(a), we display the counting distribution for the Thomas process, $p(n)$ vs $n$, with $2 T / \tau_{p}$ as a parameter, when $\alpha=0.5$ and $\langle N(T)\rangle=10$. In Fig. 5(b), the case for $\alpha=2$ is shown. In both cases, the variance increases with $T / \tau_{p}$, as is understood from Fig. 4 and Eq. (37). Furthermore, for fixed $T / \tau_{p}$, the count uncertainty increases with $\alpha$, which can also be understood on the basis of Eq. (37).

In Fig. 6 we compare the Thomas and SNDP counting distributions in the limit $T / \tau_{p} \gg 1$. (In this limit the SNDP is described by the Neyman type- $A$ counting distribution.) In Fig. 6(a) we present the results for identical values of $\mu T(=5)$ and $\alpha(=1)$ in both cases. Clearly the mean and variance for the Thomas (10 and 25 , respectively) are greater than that for the Neyman type- $A$ ( 5 and 10 , respectively) because of the feedforward path. In Fig. 6(b), we effect the comparison on the basis of an identical overall count mean $[\langle N(T)\rangle=$ 10] for $\alpha=2$. (Thus the driving rate $\mu$ differs in the two cases.) The most pronounced differences should appear for moderate values of $\alpha$ since the Thomas counting distribution becomes the Poisson when $\alpha=0$, and the SNDP approaches the Poisson when $\alpha \rightarrow 0 .{ }^{11}$ Also, in the limit of large multiplication parameter $(\alpha \rightarrow \infty)$, the SNDP and the Thomas counting distributions both approach the fixed multiplicative Poisson distribution. ${ }^{11}$ Even for $\alpha=2$, however, it is apparent from Fig. 6(b) that the distributions for the two cases are quite similar, although the variance for the Thomas is slightly greater. Furthermore, for $\beta \ll 1$, the Fano factor for the SNDP, $F_{\mathrm{SNDP}}$, has been shown to approach 1 when $\beta$ is also $\ll 1 / \alpha .^{6}$ For the Thomas, $F_{T}$ approaches 1 when $\beta$ is also $\ll(1+\alpha) / \alpha$. 

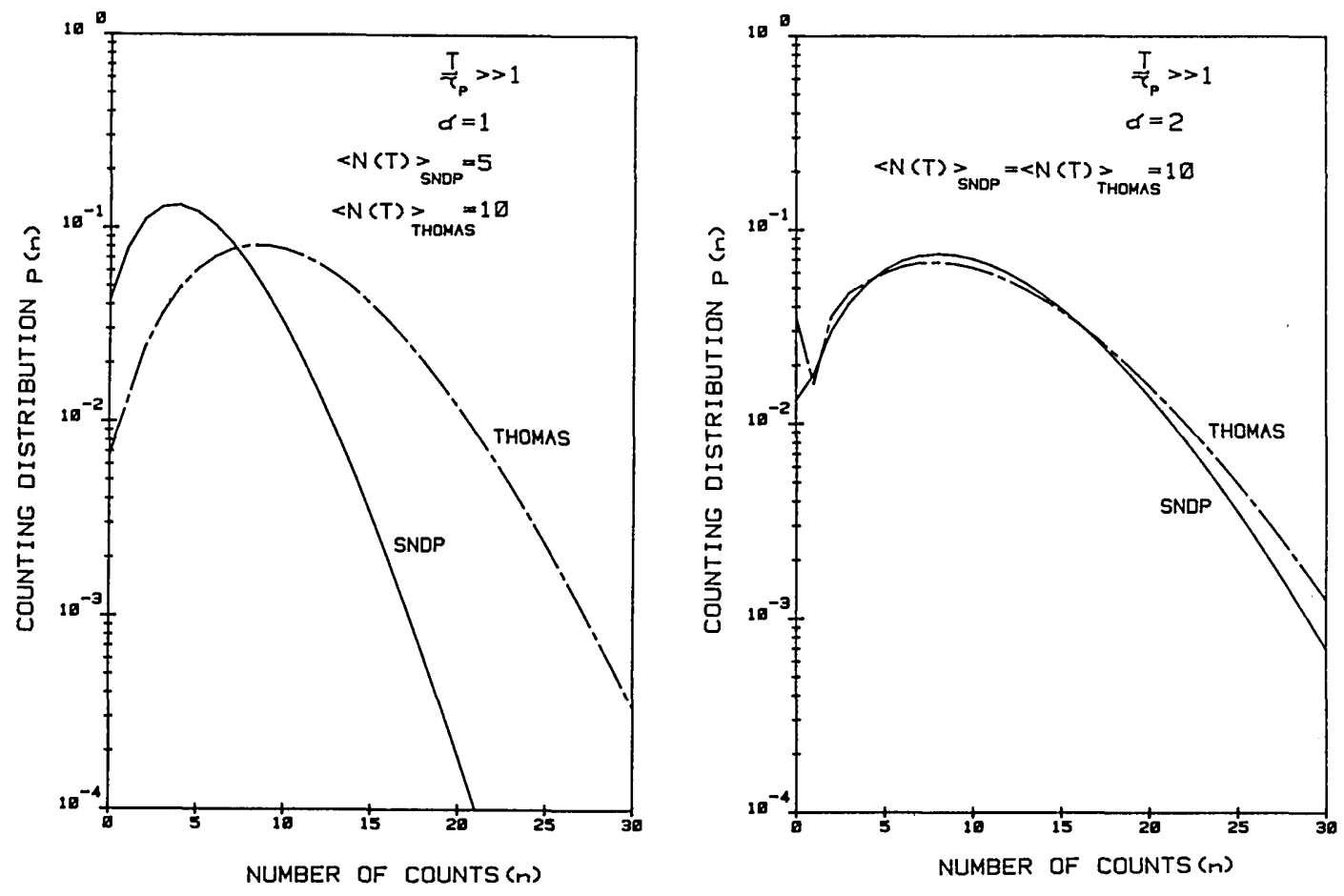

(a)

(b)

Fig. 6. Thomas and SNDP counting distributions, $p(n)$ vs count number $n$, in the limit $T / \tau_{p} \gg 1$. In this limit, the SNDP reduces to the Neyman type-A distribution. (a) $\mu T=5$ and $\alpha=1$ for both cases, so that $\langle N(T)\rangle_{\mathrm{SNDP}}=5$ and $\langle N(T)\rangle_{\mathrm{Thomas}}=10$; (b) $\alpha=2$ and $\langle N(T)\rangle_{\mathrm{SNDP}}$ $=\langle N(T)\rangle_{\text {Thomas }}=10$ for both cases.

\section{E. Counting Correlation Function}

In this subsection, we obtain the correlation function between counts observed in two time intervals of duration $T$, separated by a time delay $\tau=t_{2}-t_{1}$, for the Thomas process. The autocorrelation function is defined as ${ }^{2}$

$$
R\left(t_{1}, t_{2}\right)=\left\langle\left[N\left(t_{1}+T\right)-N\left(t_{1}\right)\right]\left[N\left(t_{2}+T\right)-N\left(t_{2}\right)\right]\right\rangle .
$$

It is obtained from the 2-D mgf expressed in Eq. (31) by setting $L=2, T_{1}=T_{2}=T$, and by forming the derivative

$$
R\left(t_{1}, t_{2}\right)=\left.\frac{\partial}{\partial s_{1}} \frac{\partial}{\partial s_{2}} Q_{N}(\mathbf{s})\right|_{\mathbf{s}=0} .
$$

A straightforward calculation yields the autocorrelation function for the Thomas process:

$$
\begin{aligned}
R(\tau)= & \langle N(T)\rangle^{2}+\langle N(T)\rangle \phi(\tau), \\
\phi(\tau)= & \left(1-\frac{|\tau|}{T}\right) u(T-|\tau|)+\frac{1}{1+\alpha}\left\{\frac{1}{T} \int_{-\infty}^{\infty} h_{T}(x) h_{T}(x-\tau) d x\right. \\
& \left.+\int_{-T}^{T}\left(1-\frac{|x|}{T}\right)[h(x-\tau)+h(\tau-x)] d x\right\}, \tau \neq 0, \\
\phi(0)= & \frac{1}{1+\alpha}\left(1+\frac{3 \alpha}{M^{\prime}}+\frac{\alpha^{2}}{M}\right),
\end{aligned}
$$

where

$$
\tau=t_{2}-t_{1}, \quad u(x)= \begin{cases}1 & x \geq 0, \\ 0 & x<0,\end{cases}
$$

and $\mathcal{M}, \mathcal{M}^{\prime}$ are provided in Eqs. (35a) and (35b), respectively.
For the usual example of an exponential impulse response function, it can be shown that

$$
\phi(\tau)=\left\{\begin{array}{cc}
\frac{1+3 \alpha+\alpha^{2}}{1+\alpha}\left(1-\frac{|\tau|}{T}\right)+\frac{\alpha(2+\alpha)}{(1+\alpha)}\left(\frac{1}{2 \beta}\right) \\
\times\left[\exp (-2 \beta) \cosh \left(2|\tau| / \tau_{p}\right)-\exp \left(-2|\tau| / \tau_{p}\right)\right], \\
|\tau| \leq T, \\
\alpha \frac{2+\alpha}{1+\alpha} b \exp \left(-2|\tau| / \tau_{p}\right) & |\tau| \geq T,
\end{array}\right.
$$

with

$$
\begin{aligned}
& b=\frac{\cosh (2 \beta)-1}{2 \beta}, \\
& \beta=T / \tau_{p} .
\end{aligned}
$$

The counting correlation function is, therefore, exponential. The result may be compared with that derived for the SNDP. ${ }^{8}$ It is clear that they are quite similar, converging to the same result for $\alpha \gg 1$, as they should.

\section{F. Time Statistics}

We now determine the statistics for the forward recurrence time and the interevent time for the Thomas process. The forward recurrence time is defined as the time to the first event from an arbitrary time instant. This may be mathematically expressed as ${ }^{2,22}$

$P_{1}(\tau)=\lim _{\Delta \tau \rightarrow 0} \frac{1}{\Delta \tau} \operatorname{Prob}[0$ events in $(t, t+\tau)$,

1 event in $(t+\tau, t+\tau+\Delta \tau)]$. 
Since the process is taken to be stationary, we set $t=0$ in Eq. (57): To calculate the above joint probability, we make use of the probability generating function. Using the substitutions

$$
\begin{aligned}
L & =2, \\
v_{1}(t) & =u(t)-u(t-\tau), \\
v_{2}(t) & =u(t-\tau)-u(t-\tau-\Delta \tau),
\end{aligned}
$$

in Eq. (31) yields the 2-D joint mgf

$$
\begin{aligned}
Q_{\mathrm{N}}(\mathrm{s})= & \exp \left\{\mu \int _ { - \infty } ^ { \infty } \left[\operatorname { e x p } \left(-\sum_{j=1}^{2} s_{j} v_{j}(t)+\int_{-\infty}^{\infty} h(\sigma-\tau)\right.\right.\right. \\
& \left.\left.\left.\times\left\{\exp \left[-\sum_{j=1}^{2} s_{j} v_{j}(\sigma)\right]-1\right\} d \sigma\right)-1\right] d t\right\} .
\end{aligned}
$$

The probability generating function is related to the result in Eq. (58) by means of

$$
G_{\mathbf{N}}(\mathbf{z})=\left.Q_{\mathbf{N}}(\mathbf{s})\right|_{\mathbf{s}=-\ln \mathbf{z}}
$$

Because of the stationarity, we set $t=0$ in Eq. (62) as before. The 3-D joint probability can be found by the following substitutions in Eq. (31):

$$
\begin{aligned}
L & =3, \\
v_{1}(t) & =u(t)-u\left(t-\Delta \tau_{1}\right), \\
v_{2}(t) & =u\left(t-\Delta \tau_{1}\right)-u\left(t-\Delta \tau_{1}-\tau\right), \\
v_{3}(t) & =u\left(t-\Delta \tau_{1}-\tau\right)-u\left(t-\Delta \tau_{1}-\tau-\Delta \tau_{2}\right),
\end{aligned}
$$

so that

$$
\begin{aligned}
Q_{\mathrm{N}}(\mathbf{s})= & \exp \left\{\mu \int _ { - \infty } ^ { \infty } \left[\operatorname { e x p } \left(-\sum_{j=1}^{3} s_{j} v_{j}(t)+\int_{-\infty}^{\infty} h(\sigma-t)\right.\right.\right. \\
& \left.\left.\left.\times\left\{\exp \left[-\sum_{j=1}^{3} s_{j} v_{j}(\sigma)\right]-1\right\} d \sigma\right)-1\right] d t\right\} .
\end{aligned}
$$

The 3-D joint probability generating function is related to the 3-D joint mgf by Eq. (59), and the joint probability is obtained as

$$
\begin{gathered}
\text { Prob }\left[1 \text { event in }\left(0, \Delta \tau_{1}\right), 0 \text { events in }\left(\Delta \tau_{1}, \tau+\Delta \tau_{1}\right) ; \quad 1 \text { event in }\left(\tau+\Delta \tau_{1}, \tau+\Delta \tau_{1}+\Delta \tau_{2}\right)\right] \\
=\left.\frac{\partial}{\partial z_{1}} \frac{\partial}{\partial z_{3}} G_{N}(\mathbf{z})\right|_{\mathbf{z}=0} .
\end{gathered}
$$

where

$$
\begin{aligned}
\mathrm{z} & =\left(z_{1}, z_{2}\right), \\
\ln \mathrm{z} & =\left(\ln z_{1}, \ln z_{2}\right) .
\end{aligned}
$$

From the definition of the probability generating function, we have

$$
=\left.\frac{\partial}{\partial z_{2}} G_{N}(z)\right|_{z=0}
$$$$
\text { Prob[0 events in }(0, \tau), \quad 1 \text { event in }(\tau, \tau+\Delta \tau)]
$$

Combining Eqs. (58), (59), and (60), it can be shown that the forward recurrence time probability density has the following form:

$$
\begin{aligned}
P_{1}(\tau)= & \mu\left\{1+\int_{0}^{\infty} \exp \left[-h_{\tau}(t)\right] h(\tau+t) d t\right\} \\
& \times \exp \left[-\mu\left(\tau-\int_{0}^{\infty}\left\{\exp \left[-h_{\tau}(t)\right]-1\right\} d t\right)\right],
\end{aligned}
$$

where

$$
h_{\tau}(t)=\int_{0}^{\tau} h\left(t+t^{\prime}\right) d t^{\prime} .
$$

The interevent time density is the probability density function for the time intervals between consecutive events. This is the same as the conditional joint probability that a single event occurs in $\left(t, t+\Delta \tau_{1}\right)$, zero events occur in $\left(t+\Delta \tau_{1}, t+\tau+\Delta \tau_{1}\right)$, and a single event occurs in $\left(t+\tau+\Delta \tau_{1}, t+\tau+\Delta \tau_{1}+\Delta \tau_{2}\right)$, conditioned on the occurrence of a single event in $\left(t, t+\Delta \tau_{1}\right) .^{2,22}$ Using the definition of conditional probability, ${ }^{24}$ we express this as
Similarly,

$$
\operatorname{Prob}\left[1 \text { event in }\left(0, \Delta \tau_{1}\right)\right]=\left.\frac{\partial}{\partial z_{1}} G_{N}(z)\right|_{\mathbf{z}=0} .
$$

Combining Eqs. (62)-(65), the interevent time probability density turns out to be $\mathrm{be}^{25}$

$$
\begin{aligned}
P_{2}(\tau)= & \frac{1}{1+\alpha} \exp \left(\mu \tau-\mu \int_{0}^{\infty}\left\{\exp \left[-h_{\tau}(t)\right]-1\right\} d t\right) \\
& \times\left[h(\tau) \exp \left[-h_{\tau}(0)\right]\right. \\
& +h(-\tau)+\int_{0}^{\infty} h(t) h(t+\tau) \exp \left[-h_{\tau}(t)\right] d t \\
& +\mu\left\{1+\int_{0}^{\infty} h(t+\tau) \exp \left[-h_{\tau}(t)\right] d t\right\} \\
& \left.\times\left\{\exp \left[-h_{\tau}(0)\right]+\int_{0}^{\infty} h(t) \exp \left[-h_{\tau}(t)\right] d t\right\}\right],
\end{aligned}
$$

where

$$
h_{\tau}(t)=\int_{0}^{\tau} h\left(t+t^{\prime}\right) d t^{\prime} .
$$

For the exponential filter example, we plot $P_{1}(\tau)$ and $P_{2}(\tau)$ vs $\tau$ for different values of $\alpha$ and $\tau_{p} / 2$ when $\langle\tau\rangle$ $=1$ in Figs. 7 and 8. Figure 7 represents curves for $P_{1}(\tau)$, where $\tau$ is the forward recurrence time, whereas Fig. 8 represents curves for $P_{2}(\tau)$, where $\tau$ is the interevent time. When $\alpha$ is very small, both densities, $P_{1}(\tau)$ and $P_{2}(\tau)$, are approximately exponential. As $\alpha$ increases, the densities become skewed toward the $\tau=$ 0 axis, which is a manifestation of bunching. When the mean time interval and $\alpha$ are held fixed, decreasing $\tau_{p} / 2$ similarly skews the densities toward the $\tau=0$ axis, as

$P_{2}(\tau)=\lim _{\substack{\Delta, \tau_{1} \rightarrow 0 \\ \Delta r_{2} \rightarrow 0}} \frac{1}{\Delta \tau_{1} \Delta \tau_{2}} \operatorname{Prob}\left[1\right.$ event in $\left(t, t+\Delta \tau_{1}\right), 0$ events in $\left(t+\Delta \tau_{1}, t+\tau+\Delta \tau_{1}\right), 1$ event in $\left.\left(t+\tau+\Delta \tau_{1}, t+\tau+\Delta \tau_{1}+\Delta \tau_{2}\right)\right]$

$$
\lim _{\Delta \tau_{1} \rightarrow 0} \frac{1}{\Delta \tau_{1}} \operatorname{Prob}\left[1 \text { event in }\left(t, t+\Delta \tau_{1}\right)\right]
$$




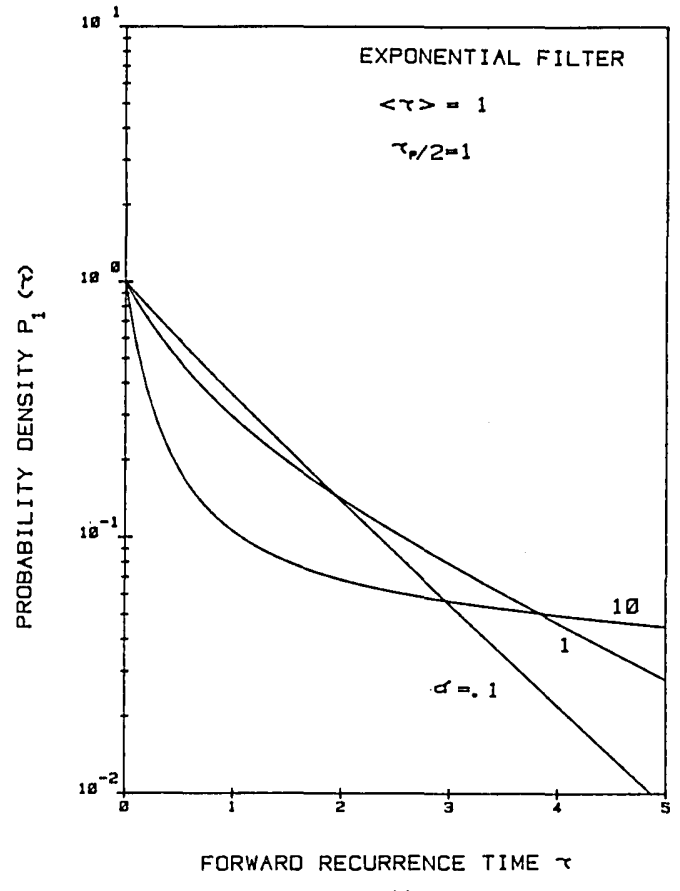

(a)

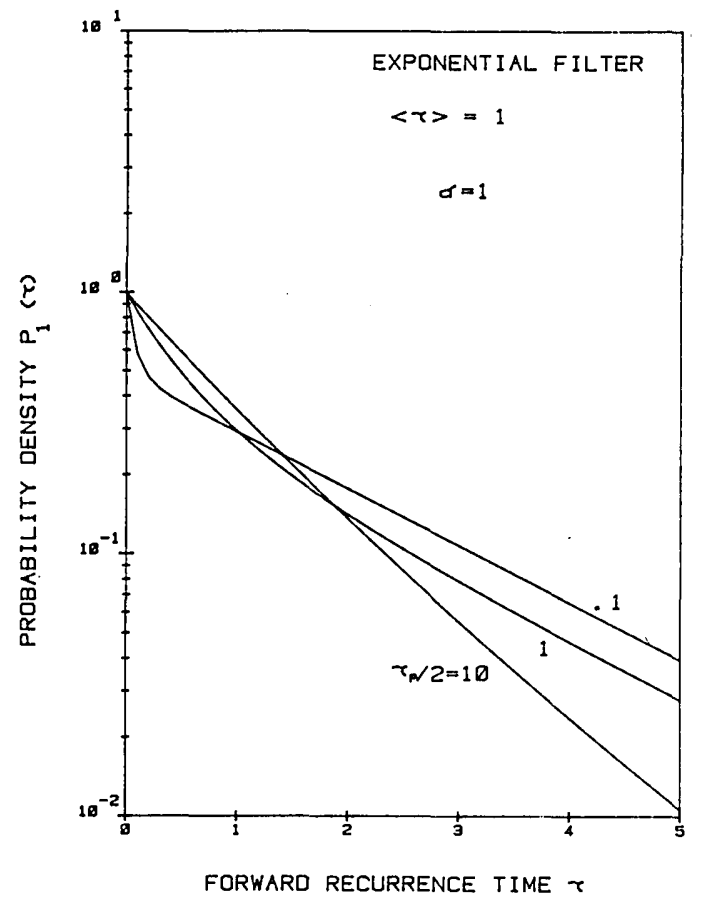

(b)

Fig. 7. Forward recurrence time probability density function $P_{1}(\tau)$ for the Thomas process. The impulse response function for the filter is exponential with time constant $\tau_{p} / 2$. In all cases the mean forward recurrence time is $\langle\tau\rangle=1$. (a) Filter time constant $\tau_{p} / 2=1$, multiplication parameter $\alpha=0.1,1,10$; (b) $\alpha=1, \tau_{p} / 2=0.1,1,10 . P_{1}(0)$ is always equal to $1 /\langle\tau\rangle$.

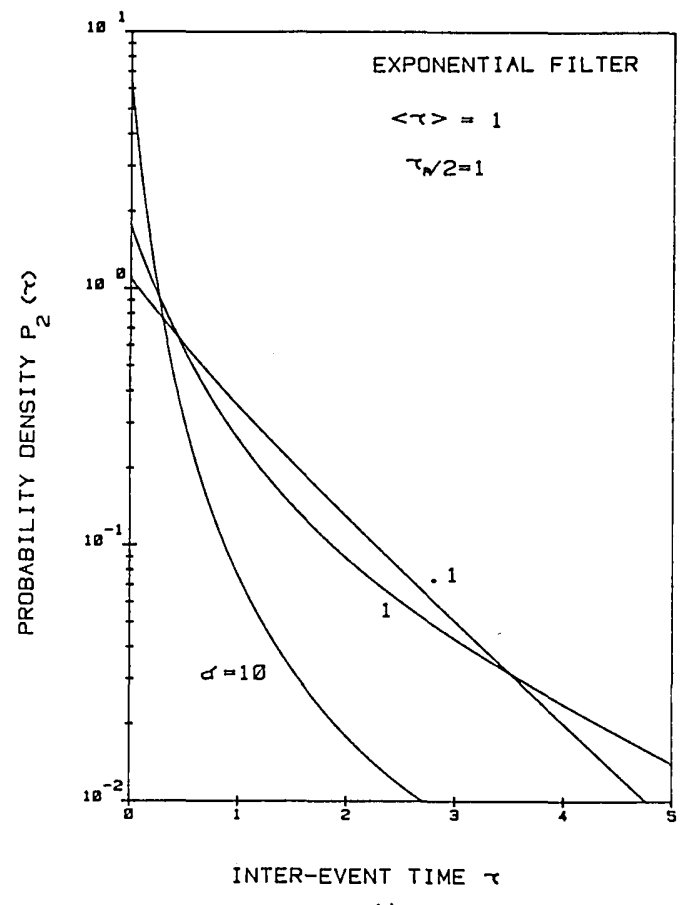

(a)

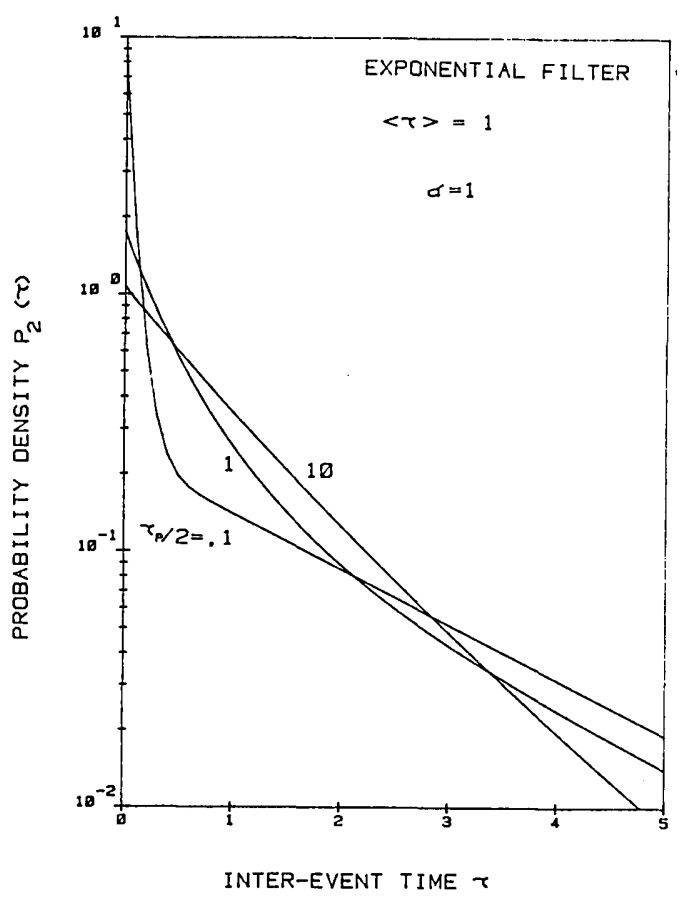

(b)

Fig. 8. Interevent (pulse-interval) probability density function $P_{2}(\tau)$ for the Thomas process. The impulse response function for the filter is exponential with time constant $\tau_{p} / 2$. In all cases the mean interevent time is $\langle\tau\rangle=1$. (a) Filter time constant $\tau_{p} / 2=1$, multiplication parameter $\alpha=0.1,1,10$. (b) $\alpha=1, \tau_{p} / 2=0.1,1,10$. 
is evident from the figures. The results are very similar to those for the SNDP presented in Figs. 10 and 11 of Ref. $8 .{ }^{25}$

\section{Applications of the Thomas Process}

The mathematical description discussed to this point applies to many physical and optical phenomena. The Thomas model will be useful in problems similar to those characterized by the SNDP $8,11,12,14$ when primary events are included in the final point process. These include $x$-ray radiography, tomography, electronography, and image intensification. Of course, in those cases where the primary and/or secondary events are not describable by a Poisson process, the Thomas process will not provide proper representation. We briefly consider a number of applications of special interest in optics. In particular, we use the Thomas model for describing the detection of scintillation and Cherenkov photons created by nuclear particles and photomultiplier noise induced by ionizing charged particles.

\section{A. Scintillation/Cherenkov Photon Counting}

The detection of ionizing radiation is often accomplished through a radiation-matter interaction in which a single high-energy particle produces a shower of particles of lower energy. A case in point is the scintillation detector, which is a combination of a scintillation crystal (e.g., NaI:Tl, plastic) with a photomultiplier tube. ${ }^{26}$ Conditions for the validity of the SNDP in describing scintillation detection are that the incident primary ionizing particles (e.g., electrons, gammas, protons) be representable as a homogeneous Poisson point process and that each primary event have associated with it an impulse response function $h(t)$ that directly governs the rate of production of Poisson optical photons. ${ }^{6,8,11,14}$

When the primary process consists of high-energy charged particles (e.g., electrons), Cherenkov radiation can be produced in addition to luminescence radiation. However, if a large number of photoelectrons are generated by the Cherenkov photons arising from a single particle, they will appear as a single (large) photoelectron pulse, since the Cherenkov radiation emission time is much shorter than the transit time in the photomultiplier. In the presence of Cherenkov radiation, therefore, the (unmarked) overall point process will include the primary as well as the subsidiary events.

A model for this process is illustrated in Fig. 9. It is seen to be identical in form to the block diagram presented in Fig. 3, so that the resulting photon emissions form a Thomas process. The function $h(t)$ will often be approximately a decaying exponential, so that the counting and time statistics are given by Eqs. (51) and Eqs. (61) and (66), respectively. The description is completely characterized by $\mu$ and $h(t)$ and, therefore, in this case, by three parameters: $\mu$, the rate of the primary process; $\alpha$, the multiplication parameter; and $\tau_{p}$, the lifetime of the process of secondary event generation. If we perform photon counting, we must adjoin $T$, the counting time. The Thomas process provides a realistic way of incorporating the effects of finite quadrat size, as does the SNDP. ${ }^{8}$

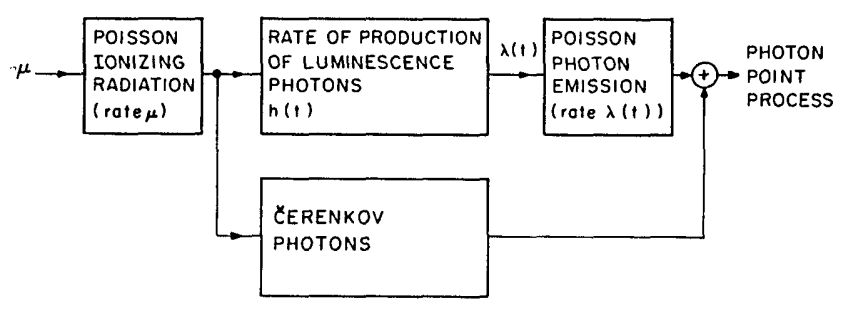

Fig. 9. Model for the photon point process generated by betaluminescence photons plus single Cherenkov photon bursts. Observe the relation to Fig. 3, which is the mathematical model studied here.

It is clear that in the limit of counting times much longer than the exponential decay time, the counting distribution will reduce to the Thomas probability distribution for arbitrary $h(t)$. The special mathematical properties of this distribution, such as permanence under convolution and convergence in distribution to the Gaussian, have been discussed elsewhere. ${ }^{11}$ It is interesting to note that the fits provided by the Neyman type- $A$ distribution to the (large $T$ ) experimental data reported in Refs. 6,8 , and 14 are always as good as or better than those provided by the Thomas distribution.

\section{B. Photomultiplier Noise Induced by lonizing Radiation}

In certain applications in which we wish to observe photon arrivals by using a photomultiplier tube, e.g., in astronomy conducted at high altitudes or in space, the description provided above may be characteristic of the noise rather than of the signal. Viehmann and Eubanks ${ }^{27,28}$ have discussed sources of noise in photomultiplier tubes in the ionizing radiation environment of space. Such noise may arise from several mechanisms such as luminescence and Cherenkov radiation in the photomultiplier window; secondary electron emission from the window, photocathode, and dynodes; bremsstrahlung in turn causing such secondary electron emission; cosmic-ray bursts; and, of course, thermionic emission dark current. These effects clearly degrade both the dynamic range and the photometric accuracy of low-light-level measurements and, therefore, must be properly modeled. It is evident from the experimental results reported in the previous subsection that the SNDP and Thomas processes provide a sound point of departure in modeling a number of these sources of noise.

\section{v. Conclusion}

Thorough consideration has been given to the counting and time statistics for the Thomas process. It provides a natural description for phenomena involving the random multiplication (or reduction) of Poisson point events with a random time delay, when primary events are included in the overall point process. The model has application in a number of important problems in electrical engineering, physics, and optics, including photomultiplier-tube luminescence and Cherenkov noise induced by ionizing radiation, the photon counting detection of nuclear particles, x-ray radiography, tomography, and electronography. There are 
other single-stage random multiplication processes involving random delay, for which this model provides a ready solution.

The model could be extended and strengthened in a number of ways. In this concluding section, we point to various restrictions that have been implicitly and explicitly imposed on the mathematical formulation and discuss ways in which these restrictions can be relaxed.

The primary Poisson point process was taken to be homogeneous (HPP) and, therefore, stationary (see Fig. 3). Physical situations in which primary events are nonstationary also occur, and it is of interest to determine the statistical properties of the resultant process. Such an analysis has been carried out for the nonstationary SNDP, where primary events are excluded. ${ }^{12}$ Similarly, the nature of the process in the presence of interference effects ${ }^{15}$ remains to be worked out. We should point out that, unlike the DSPP (and the SNDP), ${ }^{20}$ the Thomas process is not invariant under random deletion.

Concerning the linear filter in Fig. 3, we have tacitly assumed throughout that $h(t)$ is a deterministic impulse response function. Gilbert and Pollak ${ }^{29}$ have shown that if the filter $h(t)$ contains a random parameter, an equivalent completely deterministic impulse response function $h^{\prime}(t)$ can always be found that generates $X(t)$ with identical statistics. In particular, if $h(t)=$ $h_{0}\left(t / \tau_{p}\right)$, where $\tau_{p}$ is random, $h^{\prime}(t)=h_{0}\left(t /\left\langle\left|\tau_{p}\right|\right\rangle\right)$. This is an important result because it tells us that the analysis we have carried out applies also when the linear filter is not deterministic.

Again examining Fig. 3, we see that the shot noise $X(t)$ provides the rate for the second Poisson process. Our model can be modified to permit the process to be self-excited, thereby allowing for aftereffects triggered by past events. ${ }^{2}$ This modification will be immediately useful for calculating the effects of phenomena such as dead time (absolute refractoriness) and sick time (relative refractoriness). We have carried out some of these calculations for the SNDP.7,14

Given a Thomas process (see Fig. 3), we can easily account for the effects of a statistically independent Poisson point process representing, for example, broadband background light in a photomultiplier tube. The counting statistics for the superposition process can be simply determined by numerical convolution. It should also be mentioned that the statistical behavior of the clustered processes considered here is sufficiently unique that one may conceive of innovative signal processors and receivers specifically designed to enhance such signals (or discriminate against such noise). Calculations of the performance characteristics of such devices (detection, discrimination, estimation) will have to be carried out to ascertain the value of any such proposed scheme. In the meantime, we have experimentally shown that dead time does selectively discriminate against such clusters for the SNDP.7,14

Finally, we should say a few words about higher-order clustering processes, in which each stage of a Thomas process cascades with another stage and so on. We have determined that such a cascade of Thomas processes is equivalent to an $m$-stage branching process with Poisson multiplication and arbitrary time dynamics. We shall shortly report on this work, showing that such a model provides a useful generalization of the Yule-Furry birth process. ${ }^{30}$ The results are expected to find application in characterizing the properties of devices such as electron multipliers and avalanche photodiodes. ${ }^{11}$

This work was supported by the Joint Services Electronics Program (U.S. Army, U.S. Navy, and U.S. Air Force) under contract DAAG29-82-K-0080.

\section{Appendix: Derivation of the Moment Generating Functional $L_{z}(s)$}

Let $Z(t)$ be a point process decomposable into two point processes $U(t)$ and $Z_{p}(t)$. Let $U(t)$ be a DSPP, whose rate is the process $Z_{p}(t)$ filtered by the impulse response function $h(t)$. The moment generating functional for the process $Z(t)$ is defined by 2,22

$$
L_{Z}(s)=\left\langle\exp \left[-\int_{-\infty}^{\infty} Z(t) s(t) d t\right]\right)
$$

Using the method of conditioning, the above equation can be rewritten as

$$
\left.L_{Z}(s)=\left\langle\exp \left[-\int_{-\infty}^{\infty} Z_{p}(t) s(t) d t\right]\left|\exp \left[-\int_{-\infty}^{\infty} U(t) s(t) d t\right]\right| F^{Z_{p}}\right\rangle\right)
$$

where $\mathcal{F}^{Z_{p}}$ denotes all the past history of $Z_{p}(t)$. The inner expectation can be shown to be expressible as ${ }^{16}$

$$
\begin{aligned}
\left.\left|\exp \left[-\int_{-\infty}^{\infty} U(t) s(t) d t\right]\right| \mathcal{F}^{Z_{p}}\right\rangle= & \exp \left(\int_{-\infty}^{\infty} Z_{p}(t) \int_{-\infty}^{\infty} h(\tau-t)\right. \\
& \times\{\exp [-s(\tau)]-1\} d \tau d t) .
\end{aligned}
$$

Inserting Eq. (A3) into Eq. (A2) gives

$$
\begin{aligned}
L_{Z}(s)= & \left\langle\operatorname { e x p } \left[-\int_{-\infty}^{\infty} Z_{p}(t)\left(s(t)-\int_{-\infty}^{\infty} h(\tau-t)\right.\right.\right. \\
& \times\{\exp [-s(\tau)]-1\} d \tau) d t]) \\
= & L_{Z_{p}}\left(s(t)-\int_{-\infty}^{\infty} h(\tau-t)\{\exp [-s(\tau)]-1\} d \tau\right),
\end{aligned}
$$

where the moment generating functional for the first stage is ${ }^{16}$

$$
L_{Z_{p}}(s)=\exp \left(\mu \int_{-\infty}^{\infty}\{\exp [-s(t)]-1\} d t\right)
$$




\section{References}

1. E. Parzen, Stochastic Processes (Holden-Day, San Francisco, 1962).

2. D. L. Snyder, Random Point Processes (Wiley-Interscience, New York, 1975).

3. D. R. Cox, J. R. Stat. Soc. B 17, 129 (1955).

4. M. S. Bartlett, Biometrika 51, 299 (1964).

5. A. J. Lawrance, "Some Models for Stationary Series of Univariate Events," in Stochastic Point Processes: Statistical Analysis, Theory, and Applications, P. A. W. Lewis, Ed. (Wiley-Interscience, New York, 1972), pp. 199-256.

6. M. C. Teich and B. E. A. Saleh, Phys. Rev. A 24, 1651 (1981).

7. M. C. Teich and B. E. A. Saleh, J. Opt. Soc. Am. 71, 771 (1981).

8. B. E. A. Saleh and M. C. Teich, Proc. IEEE 70, 229 (1982).

9. J. Neyman, Ann. Math. Stat. 10, 35 (1939).

10. J. Neyman and E. L. Scott, J. R. Stat. Soc. B 20, 1 (1958).

11. M. C. Teich, Appl. Opt. 20, 2457 (1981).

12. B. E. A. Saleh and M. C. Teich, "Statistical properties of a nonstationary Neyman-Scott cluster process," IEEE Trans. Inf. Theory, to be published (November 1983).

13. G. Vannucci and M. C. Teich, J. Opt. Soc. Am. 71, 164 (1981).

14. B. E. A. Saleh, J. T. Tavolacci, and M. C. Teich, IEEE J. Quantum Electron. QE-17, 2341 (1981).

15. B. E. A. Saleh, D. Stoler, and M. C. Teich, Phys. Rev. A 27, 360 (1983).

16. K. Matsuo, B. E. A. Saleh, and M. C. Teich, J. Math. Phys. 23, 2353 (1982).

17. M. Thomas, Biometrika 36, 18 (1949).

18. R. E. Burgess, Discuss. Faraday Soc. 28, 151 (1959).

19. L. Mandel, Br. J. Appl. Phys. 10, 233 (1959).

20. M. C. Teich and B. E. A. Saleh, Opt. Lett. 7, 365 (1982).

21. A. van der Ziel, Noise in Measurements (Wiley-Interscience, New York, 1976).

22. B. E. A. Saleh, Photoelectron Statistics (Springer, New York, 1978).

23. D. Gross and C. M. Harris, Fundamentals of Queuing Theory (Wiley, New York, 1974).

24. A. Papoulis, Probability, Random Variables, and Stochastic Processes (McGraw-Hill, New York, 1965).

25. The analogous result for the SNDP, represented in Eq. (49) of Ref. 8 , is incorrect. The correct result is

$$
\begin{aligned}
P_{2}^{\mathrm{SNDP}}(\tau)= & \frac{1}{\alpha}\left\{\mu \int_{-\infty}^{\infty} h(t) \exp \left[-h_{\tau}(t)\right] d t \cdot \int_{-\infty}^{\infty} h(t+\tau)\right. \\
& \times \exp \left[-h_{\tau}(t)\right] d t \\
& \left.+\int_{-\infty}^{\infty} h(t) h(t+\tau) \exp \left[-h_{\tau}(t)\right] d t\right\} \\
& \cdot \exp \left(\mu \int_{-\infty}^{\infty}\left\{\exp \left[-h_{\tau}(t)\right]-1\right\} d t\right) .
\end{aligned}
$$

The graphical result for the SNDP, presented in Fig. 11 of Ref. 8 , is correct, however.

26. J. B. Birks, The Theory and Practice of Scintillation Counting (Pergamon, Elmsford, N.Y., 1964).

27. W. Viehmann and A. G. Eubanks, "Noise Limitations of Multiplier Phototubes in the Radiation Environment of Space," NASA Tech. Note D-8147 (Goddard Space Flight Center, Greenbelt, Md., Mar. 1976).

28. W. Viehmann, A. G. Eubanks, F. G. Pieper, and J. H. Bredekamp, Appl. Opt. 14, 2104 (1975).

29. E. N. Gilbert and H. O. Pollak, Bell Syst. Tech. J. 39, 333 (1960).

30. K. Matsuo, M. C. Teich, and B. E. A. Saleh, "Poisson branching point processes," in preparation.
Books continued from page 1892

In Part 4 we find Pattern Recognition, e.g., statistics and memory networks. Part 5 presents Spatially Analog Processes such as the use of (conceptually) elastic templates; Part 6 Higher Level Representations in Image Processing, which includes the hierarchical sequence of computer operations and the role of attention in object perception and cognition.

Where do we go from here? Quoting from the Postscript, "the human visual system is the most powerful information-processing system known. It very rapidly processes a continuous stream of information, it copes with an enormous variety of formats, and many kinds and degrees of image degradation, and it can be used for purposes as varied as sight-reading music, peeling a banana, and enjoying a ballet." No sane person can claim to know it all. Nevertheless, we keep trying.

\section{JURGEN R. MEYER-ARENDT}

Magnetic Electron Lenses. Edited by P. W. HAWKES. Springer-Verlag, Heidelberg, 1982. 462 pages. $\$ 44.50$.

As underlined in the Preface, the aim of this book is to fill the lack of a textbook covering the properties of magnetic lenses. At first sight one may ask if it represents a comprehensive survey of a deep and elegant compilation through a huge amount of experimental work and reflective dissertation by experts about various aspects within the limits of a particular field (optical properties, lens design, construction and practical applications, precise references to actual experiments and results, and possible prospects). Indeed it is all of that. It seems that as it answers most questions for the layman it also brings together a mine of useful data and gives insight into some specific methods (e.g., system design), the accounts of which were scattered in scientific papers or not available; it certainly opens new vistas for those concerned with the subject.

The first chapter on Magnetic Lens Theory by P. W. Hawkes will be a regale for physicists familiar with optical design; it begins with what they will recognize as fundamental geometrical optics (Fermat's principle and stationarity of the path). Paraxial properties and cardinal elements are clearly defined as well as conjugation relationships, where from the paths of rays are derived distinct treatments for objective lenses and other components, namely, projectors for which it is necessary to consider asymptotic conditions. Geometrical and chromatic aberration coefficients are also established and discussed separately.

Since the general methods of reasoning here and in conventional optics are much alike, one could have expected some consideration of the mutual influence of the various types of aberration (at different orders) for satisfying tolerance criteria and finally how aberrations combine with field angle and numerical aperture as diffraction intervenes; concepts of spread functions and overall transfer could have been defined (e.g., from the resultant diffusion pattern).

The second chapter, Magnetic Field Calculation and the Determination of Electron Trajectories, by E. Kasper turns its demonstrations and developments to the use of the digital computer in the design of lenses, with emphasis on field calculation, giving a point of view about electron trajectories and aberrations coefficients slightly different from that in the previous chapter. It is interesting to compare the two of them. In addition, despite a clear coverage of the theory which is easily readable and good examples of applications in their final forms, it is not obvious that the balance between mathematical parts and applied engineering is entirely satisfactory; putting the methods discussed into practice would involve going back to the many papers referred to in the text. Nevertheless, it presents a survey of the relevant literature illustrated with typical curves that support well the text for which a background knowledge is necessary.

continued on page 1944 